\section{MULTIPLE CUTANEOUS GANGRENE OF THE} SCALP IN A CACHECTIC CHILD.

\section{By A. DOUGLAS HEATH, M.D.Lond.,} Birmingham.

Some details of the following case may perhaps be of interest not only because this variety of gangrene is not commonly met with, but also from the fact that it appears to differ in some particulars from the types of gangrenous dermatitis most frequently seen in infants and young children, which have been described by well-known observers under the names of varicella gangrænosa, rupia escharotica, and dermatitis gangrænosa infantum. With regard to predisposing causes in this case it would seem that whooping-cough and bronchitis were the only illnesses that the child had suffered from previous to the onset of the gangrene, and these had begun three or four weeks before the first patch of gangrene became visible on the scalp, and had evidently produced a very marked effect on the patient, as the loss of strength and weight had been rapid throughout. The gangrene when once started ran its course to a fatal termination in four weeks, but the disease was much more rapid in its spread during the last seven days of the patient's life than in the previous three weeks. The gangrenous patches were entirely confined to the hairy scalp, which in the intervening areas appeared to be perfectly healthy, and no skin lesions of any kind were present on the rest of the body. No discharge from the nose or ears could be seen, nor could I find any other source from which infection of the scalp might have taken place. There was no previous history of varicella either in the patient or in his brothers and sisters, and I examined all the children in the family for that disease with a negative result. I could also detect no signs of syphilis in the patient nor the other children, and the examination of the father and mother also revealed no evidence of that disease. There was no history of miscarriages in the latter, and all her children were living and, with the exception of the patient, healthy. Vaccination had been performed when the child was under three months of age. There was no family history of tubercle.

T. H., aged a years, was brought to me on January 1 th, 1897 . He was very emaciated. He showed no signs of rickets, and the mother said that Cere plump and healis piden rales, rales,

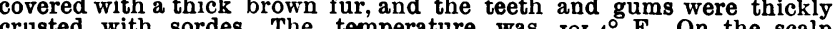
in the with sorart. in the upper part of the occipital region on the right side, as for shilling arawn he shiling piece, wlack tough, dre margin of. the ulcer was pale in colour, and seemingly somewhat actul chual the surfece of the heen.

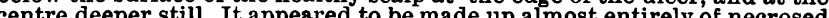
enine deeper or dry. On raising the slough with a probe a little sutery and a thist or dry. On raising the slough with a probe a little watery and slightly unhelthylooking fatty tigsue appeared to be covering the and a this, unhealthy-looking fatty tissue appeared to be covering the aponeurosis dhe deeply excavated ulcer would remind one more strongly of the appearr a case col wo purple at the edges. The centre of the purple at the edges. The centre of the gangrenous area was whole patch was gently pressed between the tips of the fingers it felt distinctly soft, and gave one the impression of fluctuating from side to side. A little behind the left parietal eminence, and nearly two inches above this last gangrenous area was a over it ras of actuang over it was of a very pale pink colour, and a few small silvery scales due to a very slight desquamiation of the epidermis hairs springing from this area were quite long and normal in appearance, and did not seem to be loosened. There was also no visible heaping up of epithelium around their bases, and no points of suppuration could be seen. This small spot had evidently been the last to appear, and had not been noticed by the child's mother before 1 drew her attention to it vihich is not surprising, so little had the superficial layers of the epidermis over this softened area altered in appearance. The uppermost of pus, and a slight swelling in this region is shown in the first drawing. No pus, and a slight swelling in this region is shown in the first dra

The description of the first appearance and early course of the gangrene as given by the child's mother is interesting. The oldest lesion of the scalp, which afterwards became the largest area of gangrene, began as a large "pimple" three weeks before the case came under my notice. I very soon, so the mother said, became exactly like the small pink swelling I had drawn her attention to on January inth, and, after further enlargement, began to subside, and shortly afterwards to turn black, and the large slough reached the size it had attained when I saw it in about ten days. During the next week the black slough had sunk deeper and deeper into the cavity of the ulcer, and had also become detached at its edges. The second gangrenous area began in exactly the same way three days after the first, and had progressed more slowly, having, as I said before, still a purple-red zone around its black centre when I first exaafter the lesions of the scalp had appeared.

During the eight days that I was enabled to watch the patient the two large gangrenous patches remained practically in statu quo, the only change being that the black centre in the smaller of the two increased in size by extension of the gangrene into the darkly coloured zone around it. The small pink fluctuating swelling behind the left parietal eminence that I noted on January Irth, at first increased in size, and on January r3th covered an area as large as a slilling, but was only very slightly raised above the level of the healthy scalp, and still retained its light pink colour. The small thin scales on the surface of the swelling were more numerous, and the hairs. unaltered in appearance, were still unloosened. A fairly rapid deepening of colour over the whole of the softened area now took place, and on January r4th this discoloration had approached almost to black in the centre around two or three hairfollicles, and at the same time this portion of the surface had commenced to sink more a slightly concave hollow had been produced. The hitherto dry surface now became more and more moist in the centre of this depressed area where the colour was deepest, but no vesication took place, and the necrosed skin, except for its black colour and sodden appearance, seemed
otherwise unaltered. On January is th the black patch in the centre

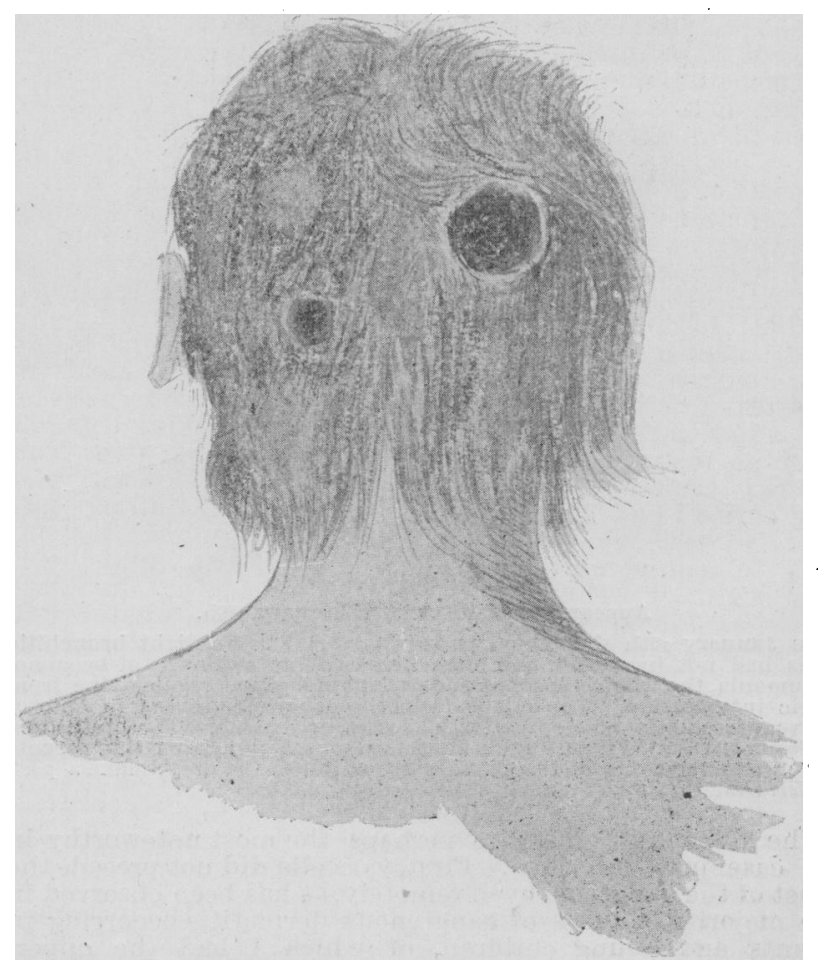

Appearance of disease on January 13th

was as large as a sixpence, and the whole area was still more deeply sunk below the level of the scalp. The hairs were now easily removed from the slough, a little clear bloodstained fluid following their removal. This black gangrenous portion had increased to the size of a shilling by coloured skin at its wases. January isth five mores. Dung in exactly the same course even more rapidily than the previous, anes had done gangrene starting in four out of the five fresh lesions before they were larger than a sixpence. None of these appeared close together, and there was quite an inch between the two nearest. No points of suppuration were visible in any of these small swellings, and the hairs did not seem to be loosened until gangrene set in. By a comparison of $\mathrm{my}$ two drawings made on January progress of the case can be seen. Three fresh gangrenous areas are shown in the second drawing, the remaining two being more on the top of the head do not appear in the view taken from behind. The seoond sketch does not show the swelling on the left side of the neck as the abscess had been opened and a quantity of pus drained off. The large the abscess had been opened and a quantity of pus drained off. The large
black slough covering the floor of thelarge ulcer when removed appeared to be made up of the whole thickness of the skin. It was about $\mathrm{I}-8 \mathrm{th}$ of an inch be made up of the whole thickness of the skin. It was about r-8th of an inch
thick at its margin. and quite double that thickness in its centre. Although 
the whole was soft and sodden with moisture, the cuticle was still adherent to the parts beneath, and those in their deepest levels seemed spongy and netlike, and the detached lower border had a ragged appearance frum its outline being broken by shreds of blood-stained tissue of varying sizes and lengths. In the other gangrenous patches no natural separation of the sloughs had taken place, but pieces of black tissue cut away from the centres of two of the patches were one-eighth of an inch or more in thickness, and appeared to the naked eze similar in structure to the large slough above described. The blood-stained appearance of the lower levels of the sloughs would give rise to the suspicion that there had been hæmorrhage into the swellings before they became gangrenous, but there was nothing outwardly in the colour of these to support such a view, and the fluid contained in them was clear, and only very faintly coloured. No attempt at scabbing was visible in any of the gangrenous areas, although five at least were either exposed to the air, or covered by dry dressings, and the black patch in the centre of each was finally as large as a sixpence. A purple-red zone, fading to pink at its edge, was visible around each black centre, except in the large ulcer, where separation of the slough had taken place, and in all but this the gangrene was still extending up to the time of the patient's death. As far as one could tell in such small areas, the spread of the gangrene peripherally was preceded deeper tissues by fluid.

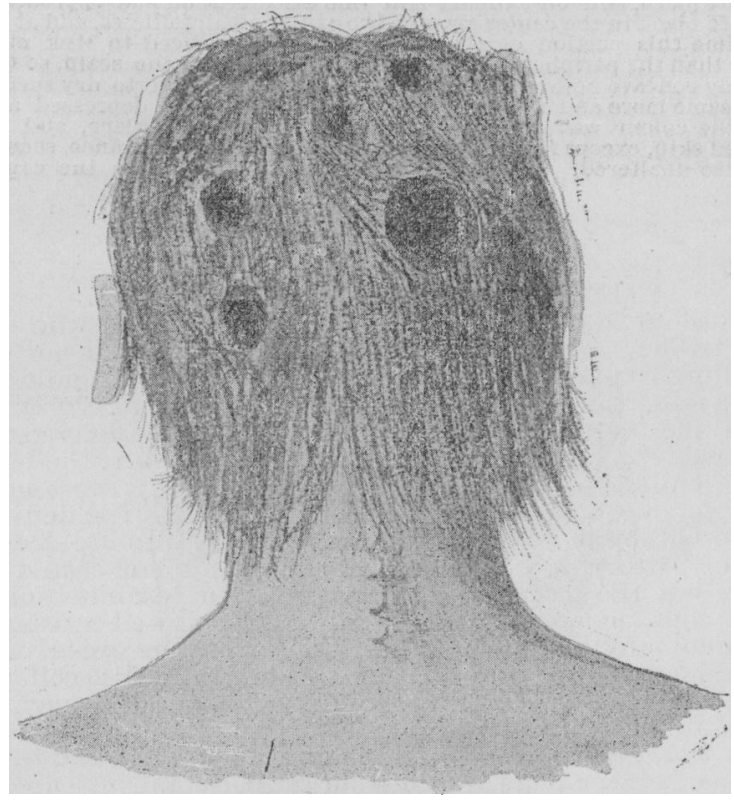

Appearance of disease on January 18 th.

On January r8th the temperature was ro ${ }^{\circ}$ F.; the slight bronchitic signs had not increased, and there was still no evidence of bronchopneumonia, the whole aspect of the patient being that of one dying from septic intoxication. The odour from the gangrenous areas, in spite of the very free use of antiseptics, was most disagreeable. The child died on January rgth. I was unable to obtain permission from the parents mortem examination.

REMARKS.

The following points are perhaps the most noteworthy in the case just described. First, varicella did not precede the onset of the gangrene, even remotely, as has been observed in the majority of cases of gangrenous dermatitis occurring in infants and young children, of which I had the opportunity of seeing a very rapidly fatal instance, through the kindness of Dr. Meredith Richards, at the Birmingham City Hospital, about eighteen months ago.

Secondly, the child had been vaccinated when under three months of age, and was just two years old when attacked with the gangrene, so that vaccinia obviously cannot have had a causal relation to it.

Thirdly, the limitation of the gangrenous areas to the scalp is an uncommon feature in cases not following either varicella or vaccinia, the lower half of the body being more frequently attacked.

Fourthly, no formation of any scab was visible on any of the gangrenous patches, although these were, in the earlier lesions, of considerable size, and in two instances had existed for three weeks, the necrosed skin remaining moist throughout, and this fact is all the more noteworthy as in the large majority of these cases of gangrenous dermatitis a scab resulting from the drying of the central portion of the gan- grenous area is formed under which ulceration still goes on in rupia-like fashion. It may be, however, that this scabbing was only prevented by an unusual amount of moisture keeping the dead skin continually saturated, and the more usual appearances of such lesions wert absent from that circumstance alone.

The mode of origin in my case was more like that seen in the formation of the multiple subcutaneous abscesses not. infrequently met with in ill-nourished children, in which the cutis over the abscess appears at first to be very little affected whilst the disease progresses in the subcutaneous fat, and the surface of the skin over quite large areas may look pale, or at the most show only a little desquamation and slight reddening. When this process had gone on for a certain time, and a slightly raised swelling covering an area as large as a sixpence or shilling had been produced, a deepening in colour, slow in the earlier but rapid in the later lesions took place, and the subsequent rapidity in the development of the gangrene in the latter seemed due to an increased virulence of the disease, and not to any accidental coneomitant like hæmorrhage into their cavities, which has been noted by Dr. Crocker ${ }^{1}$ in a few rapidly fatal cases. The large conical ulcer conformed exactly to the characteristic appearances described in most cases of gangrenous dermatitis in infants, and, as is usually the case, the spread of the gangrene stopped when the slough had become separated. The swelling and subsequent breaking down of the adjacent lymphatic gland in the neck into pus is also interesting, and I am not aware that this adenitis in the neighbouring glands has been observed in these cases before. In the absence of any material from the scalp for pathological examination one can unfortunately only surmise as to where the disease started in this particular case. The appearances were, however, all in favour of the primary focus of the mischief lying either very deeply in the skin or beneath it, as it is otherwise difficult to see why no formation of pustules or vesicles took place, and why thecutis over moderately large areas remained comparatively unaffected for so long.

Finally, the patient showed no signs of ordinary pyæmia, nor of ulcerative endocarditis; there were no rigors, the joints were not swollen, the skin remained dry, and the temperature was never higher than $102.4^{\circ} \mathrm{F}$. It would also be difficult to imagine multiple emboli from these causes producing eight distinct lesions of the scalp without the rest of the skin of the body showing any signs of similar embolic infection.

REFERENCE.

1 Diseases of the Skin, p. 277

\section{ANTISTREPTOCOCCIC SERUM IN THE TREAT-} MENT OF PUERPERAL FEVER.

By J. F. L. WHITTINGDALE, M.B., Sherborne.

I am led to report the following cases, because they show, I think, the value of the serum treatment in a case of puerperal septicæmia, and at the same time its harmlessness when used in a case which subsequently proved to be scarlet fever.

CASE 1.-Mrs. L., aged 27, primipara, was delivered on February i6th by my assistant with forceps, on account of rigidity of the perineum, which was rather badly torn and required sutures. A vaginal douche of Condy was ordered to be given thrice daily. On February 18 th, within fortyeight hours of delivery, slight rigors occurred. On February roth the temperature was ro2. $4^{\circ}$, and the pulse roo. Lochia scanty, noises in the ears, constipation, some tympanites, but no abdominal tenderness. Calomel gr. $v$ was given, and followed the next morning by ol. ricini $3 j$. which produced a copious evacuation of the bowels. On the evening of February zoth the temperature had reached $103^{\circ}$, and becameeven higher in the night, and was accompanied by delirium. On the morning of rebruary $2 \mathrm{rst}$, when I first saw her, the temperature was $102.4^{\circ}$, and the pulse $x 20$. The lochia werescanty, the wound gaping and covered with a thin grey discharge, the stitches having given way. She had had no sleep since delivery. In the evening the temperature was $103^{\circ}$, when I injected ro c.cm. of Bullock's antistreptococcic serum into the right buttock (the serum having been prepared on February 4 th). A draught of bromidia was given at bedtime, and the wound ordered to be kept stuffed with odoform gauze. The temperature two hours after the injection was ro2. $8^{\circ}$. On the 22 nd the morning temperature was ror $^{\circ}$, pulse ro8. Skin perspiring profusely. Had a better night. Rather constipated; considerable tympanites present. Had no more serum, so gave a quinine nd hydrobromic acid mixture. The evening temperature was ro ${ }^{\circ}$. On the ${ }_{23}$ rd temperature was $100.4^{\circ}$, pulse 96 in the morning. Abdomen still tympanitic; perspiration profuse, no headache, moderate amount of 